\title{
O brincar da criança com câncer no hospital: análise da produção científica'
}

\author{
Children with cancer at play in hospital: \\ analysis of scientific production
}

\author{
Adriano Valério dos Santos AZEVÊDO²
}

\begin{abstract}
Resumo
O presente artigo objetivou analisar a produção científica nacional e internacional acerca do brincar da criança com câncer no hospital. Foram realizadas consultas em bases de dados eletrônicas e capítulos de livros, para selecionar os estudos publicados no período de 2000 a 2010. Verificou-se que os estudos empíricos utilizam o método qualitativo com delineamento transversal. Essas pesquisas apontam os efeitos positivos da implantação de projetos lúdicos, aí incluída a música. Os estudos relatam as vivências da criança no brincar e as possibilidades de enfrentamento da hospitalização, além de apresentarem a perspectiva dos acompanhantes e da equipe de saúde. Nesse sentido, tais pesquisas não só orientam a assistência à criança com câncer na área da psicologia pediátrica, mas também contribuem para o entendimento de aspectos específicos do brincar no hospital, relacionados à qualidade de vida e à humanização.
\end{abstract}

Unitermos: Brincar. Criança com câncer. Hospitalização infantil.

\begin{abstract}
The aim of the present article was to analyze national and international scientific production in respect of children with cancer at play in hospital. Queries of the electronic databases and chapters from books were performed in order to select studies published in the period between 2000 and 2010. It was ascertained that the empirical studies had used the qualitative method with a cross-sectional study. The research shows the positive effects of implementing ludic projects, the experiences of children at play and the ability to cope with hospitalization, contributions related to the application of music and the perspectives of companions and the team of health professionals. The benefits of the ludic resources highlighted in the scientific studies provide guidance in helping the child with cancer in the area of pediatric psychology and contribute to the understanding of specific aspects of play in the hospital related to the quality of life and humanization.
\end{abstract}

Uniterms: Recreation. Child wild cancer. Child hospitalized.

Diante de uma doença, as crianças apresentam mudanças no contexto global do funcionamento orgânico e psicológico, desenvolvendo formas de enfrentamento quando um significado é atribuído nessa si- tuação (Valle, 2001). A vivência de uma doença crônica no universo infantil representa um aspecto relevante para investigações acerca do papel do brincar no hospital. O brincar contribui para melhorar a qualidade de

$\boldsymbol{\nabla} \mathbf{v} \boldsymbol{\nabla}$

1 Apoio: Coordenação de Aperfeiçoamento de Pessoal de Nível Superior.

2 Universidade Federal de Sergipe, Núcleo de Pós-Graduação em Psicologia Social. Cidade Universitária Prof. Aloísio de Campos, Av. Marechal Rondon, s/n., Jardim Rosa Elze, 49100-000, São Cristóvão, SE, Brasil.E-mail: <psicomais@yahoo.com.br>. 
vida da criança no período de hospitalização, amenizando as repercussões do adoecimento na esfera psíquica e na física, e atenuando os impactos negativos provenientes da ruptura do contexto sociofamiliar e dos procedimentos utilizados no tratamento.

O câncer é uma doença que se desenvolve nos genes das células, com uma capacidade de proliferação para originar tumores em áreas específicas do corpo humano (Yamaguchi, 1994). De acordo com Borges et al. (2006), câncer é uma denominação genérica, abrangendo um conjunto de doenças com múltiplas causas e alternativas de tratamento e prognóstico. As causas podem ser consideradas numa perspectiva multifatorial, englobando fatores genéticos e mudanças nos hábitos de vida dos indivíduos.

Dados epidemiológicos do Instituto Nacional do Câncer (2008) apontam a doença como a terceira causa de morte no país, um índice significativo que é atribuído ao comportamento alimentar inadequado, ao processo de envelhecimento e ao stress. Percebe-se a importância dos programas de saúde pública, com foco na prevenção dos fatores de risco da doença, e da atuação qualificada do psicólogo no acompanhamento hospitalar de pacientes com câncer - de forma específica direcionando a atenção para as crianças, visando facilitar o manejo das consequências oriundas da hospitalização e do tratamento invasivo.

As crianças hospitalizadas por período superior a cinco dias apresentam tendência para desenvolver transtornos psicológicos, dependendo das experiências anteriores de internação, do quadro clínico, do tipo de vínculo estabelecido com a família e da idade (Dias, M.N. Baptista \& A.S.D. Baptista, 2003). Chiattone (2003) destacou que, nos dois primeiros anos de vida, as crianças apresentam dificuldades para permanecer hospitalizadas, devido às características do ambiente hospitalar: paredes lisas, níveis variados de iluminação, pessoas estranhas, aparelhos específicos para a realização de exames. Esses fatores devem ser avaliados pelo psicólogo para realizar orientações junto à família e à equipe de saúde, quando a criança apresenta mudanças comportamentais secundárias a sua inserção no hospital. Além disso, o conhecimento da patologia e do prognóstico permite que estratégias de intervenção sejam utili-

566 zadas pelo psicólogo.
No hospital, as pessoas recebem o nome de pacientes, são identificadas por números e prontuários, utilizam uniformes e recebem atendimento de diversos profissionais de saúde. A criança encontra-se assustada com as mudanças que ocorrem a partir da sua inserção nesse ambiente, em virtude das regras estabelecidas pela instituição e da manipulação em seu corpo, realizada pelos profissionais do setor (Oliveira, Dias \& Roazzi, 2003). Essas ocorrências, associadas às sensações de fraqueza, representam para a criança uma ameaça a sua condição humana, e os procedimentos da equipe de saúde provocam medo e são considerados dolorosos, devido ao controle e forma de avaliação. As reações da criança no período de internação propiciam o desenvolvimento de quadros ansiosos, decorrentes da separação da família, do surgimento da patologia e da admissão no ambiente hospitalar (Lindquist, 1993). Dessa forma, é preciso refletir sobre quais estratégias podem ser utilizadas para minimizar o impacto proveniente do processo de hospitalização.

Na literatura científica, relatos de pesquisas apresentaram as contribuições das intervenções lúdicas para crianças hospitalizadas (Chiattone, 2003; Lindquist, 1993; Motta \& Enumo, 2004; Pérez-Ramos, 2006). De acordo com Carvalho e Begnis (2006), a criança no hospital percebe a ameaça de morte diante das alterações fisiológicas no seu quadro clínico, sendo necessário construir um ambiente seguro para promover de forma saudável a continuidade do ciclo evolutivo através do brincar. Acredita-se que o enfrentamento das repercussões psicológicas da doença é favorecido com a utilização do lúdico, principalmente com a abordagem em grupo, integrando as crianças e seus familiares para proporcionar momentos de satisfação e de reorganização das experiências. A criança hospitalizada com câncer necessita de uma equipe multiprofissional e de um espaço para expressar suas emoções, visando compreender a sua vivência por meio das atividades lúdicas que auxiliem a promoção da saúde integral.

O processo de hospitalização e a doença interagem na vivência da criança, sendo relevante desenvolver intervenções preventivas para minimizar as consequências provenientes da doença orgânica, as quais dificultam o tratamento e a adesão aos procedimentos necessários para sua recuperação (Ribeiro \& Angelo, 2005). Nesse contexto, a utilização do brincar no hospital 
promove uma ação terapêutica, além de auxiliar na atenção integral às necessidades da criança.

Os estudos acerca do brincar apontam a importância desse recurso para estimular as funções cognitivas e desenvolver habilidades nas crianças, por considerar que a atividade lúdica não se resume a entretenimento e diversão (Bomtempo, 1987; Bomtempo, Hussein \& Zamberlan, 1986). Antes, trata-se de uma prática educativa, que orienta a criança para o entendimento do mundo real e imaginário. Na perspectiva histórico-cultural de Leontiev (1998), o brincar representa o principal recurso para o desenvolvimento da criança nas relações sociais, o que permite facilitar a percepção de sujeito inserido no espaço social, bem como a compreensão acerca de suas atitudes por meio dos jogos simbólicos. No mesmo sentido, Vigotski (1998) estabelece correlações entre brincar, desenvolvimento e aprendizagem, evidenciando que as atividades lúdicas permitem recriar as experiências com a imaginação, facilitar a interação social, estabelecer significados acerca das ações no mundo e criar a noção de regras. As teorias da psicologia do desenvolvimento valorizam o brincar e atribuem importância para as etapas relacionadas à aprendizagem de novos papéis sociais, e, no contexto de hospitalização, isso permite que a criança perceba as possibilidades de enfrentamento e desenvolva comportamentos adaptativos.

Valle (2001) considera relevante conhecer as necessidades da criança no hospital, utilizando-se de um contato empático na relação terapêutica. O ambiente hospitalar pode representar um espaço para a criança encontrar o seu mundo natural por meio das atividades lúdicas. Mesmo diante das mudanças provocadas pelo surgimento de uma doença, o ambiente hospital pode proporcionar espaços destinados para o brincar, representando a valorização da vida e a ampliação da noção de saúde física e psíquica. A tecnologia avançada utilizada para o tratamento do câncer infantil apresenta inúmeras consequências emocionais para a criança no hospital, assim existindo a necessidade de se desenvolverem intervenções lúdicas.

Assim, tanto sob o aspecto social quanto científico, justifica-se a realização de estudos sobre a promoção do brincar no setor da oncologia pediátrica, o que permite identificar os efeitos dessa intervenção no hospital. Destaca-se a importância de investigar o quadro atual de pesquisas, para identificar perspectivas de atuação profissional e os possíveis avanços no entendimento desse objeto de estudo.

Este artigo, desenvolvido sob o viés dos aspectos psicológicos, objetivou analisar a produção científica nacional e internacional acerca do brincar da criança com câncer no hospital.

\section{Método}

Procedeu-se à revisão bibliográfica de artigos científicos indexados nas bases de dados Bireme, Bvs-Psi e Wiley Interscience, publicados no período de 2000 a 2010, com a temática do brincar da criança com câncer no hospital, o que representou o principal critério de inclusão. Utilizaram-se as palavras-chave criança com câncer, brincar no hospital, child whit cancer e play in the hospital. Foram selecionados os relatos de pesquisas em português e inglês, para apreciação dos resumos e trabalhos completos. Outras fontes utilizadas foram os capítulos de livros que apresentavam pesquisas empíricas. Após a construção do banco de dados, verificaram-se os principais aspectos evidenciados nas pesquisas (temas, participantes, metodologia e resultados), para categorização com o auxílio da análise de conteúdo (Bardin, 1979), o que possibilitou promover a descrição quantitativa e qualitativa.

\section{Resultados}

Identificaram-se 16 produções científicas (12 artigos e 4 capítulos de livros). Dos artigos, 9 eram de revistas nacionais: Acta Paulista de Enfermagem (2 artigos), Boletim Academia Paulista de Psicologia (1), Boletim de Psicologia (1), Estudos de Psicologia - Campinas (1), Revista Brasileira Crescimento e Desenvolvimento Humano (1), Revista Brasileira Saúde Materno Infantil (1), Revista da Escola de Enfermagem da USP (1) e Paidéia (1). Destacaram-se 3 artigos de revistas internacionais: Psycho-Oncology (2 artigos) e Child: Care, Health and Development (1). Com relação ao ano de publicação e frequência de artigos, obtiveram-se os seguintes dados: 2002 (1); 2003 (1), 2004 (1), 2005 (1), 2006 (1), 2007 (1), 2008 (3), 2009 (1), 2010 (2). Os capítulos de livros foram publicados com a respectiva frequência: 2006 (2) e 2010 (2). 
Sobre a metodologia das pesquisas que foram consultadas, prevaleceram os estudos qualitativos com delineamento transversal. Em relação aos participantes dos estudos, foi possível identificar crianças, acompanhantes e equipe de saúde. Após a leitura das pesquisas empíricas e da identificação das temáticas investigadas, os estudos foram divididos nas seguintes categorias: Contribuições do brincar no hospital, Vivências de crianças com câncer, Construção de projetos lúdicos e Modalidades do brincar no hospital. Buscou-se facilitar a compreensão das pesquisas por meio dessas categorias para delimitar o panorama atual das publicações.

\section{Contribuições do brincar no hospital}

As pesquisas apresentam os benefícios do brincar para a criança com câncer. Estudos experimentais apontam possíveis limitações relacionadas ao nível de stress da criança, assim como dados positivos sobre o brincar nas diferentes fases de tratamento da doença crônica. Outras pesquisas destacam as percepções de crianças, acompanhantes e profissionais de saúde acerca do brincar no hospital.

Borges, Nascimento e Silva (2008) investigaram os benefícios das atividades lúdicas para as crianças hospitalizadas com câncer, por meio de observação participante e entrevistas com crianças e acompanhantes (no caso, as genitoras). De acordo com os autores, após as intervenções lúdicas verificou-se redução das alterações de humor e comportamento das crianças. As sensações de bem-estar, a espontaneidade e o processo de socialização representaram as principais mudanças provenientes do brincar no hospital, o que repercutiu nos índices de satisfação das crianças e genitoras.

Motta e Enumo (2004) desenvolveram um instrumento composto por 41 pranchas ilustradas com cenas que correspondiam às estratégias de enfrentamento, tais como brincar, chorar e cantar. Participaram da amostra crianças hospitalizadas com câncer. Apresentadas as pranchas uma de cada vez, a criança descrevia a cena e indicava se a figura correspondia às ações por ela realizadas no hospital. O instrumento mostrou-se adequado, sendo identificada maior prevalência de estratégias facilitadoras que indicavam comportamento adaptativo das crianças. O brincar representou o principal recurso utilizado para enfrentamento da hospitalização.
Gariépy e Howe (2003) analisaram os efeitos terapêuticos do brincar para a criança com câncer, comparando com um grupo controle de crianças que não apresentavam doença crônica. Foram utilizados instrumentos quantitativos para avaliar o nível de stress e observar as reações no humor e comportamento das crianças durante o brincar. Os dados indicaram que as crianças com câncer mostraram-se menos receptivas para o brincar, principalmente nas atividades em grupo e no desempenho de papéis dramáticos. As crianças com câncer apresentaram nível mais alto de stress que as do grupo-controle, e também foi identificada a presença de temas recorrentes nas brincadeiras e a preferência pelo jogo solitário. Os autores destacaram que o stress da criança, decorrente da vivência de uma doença, reduz o interesse para o brincar; ou quando ela brinca, focaliza o isolamento, evitando a interação social e o entendimento acerca de sua experiência atual.

A pesquisa de Silva, Cabral e Christoffell (2008) procurou investigar o brincar da criança com câncer, analisando os mediadores utilizados nas brincadeiras e jogos. Foram elaboradas atividades lúdicas, com as crianças solicitadas para participação coletiva, com o propósito de construir uma produção artística e compartilhar com o grupo os significados atribuídos. A análise de discurso destacou dois temas: (1) instrumentos e signos utilizados nas brincadeiras e jogos, que relacionam os tipos de brincadeiras e o interesse nessas atividades; e (2) interação social, que integra pessoas e ambientes, com a construção de cenários para a realização das brincadeiras. As crianças demonstraram as habilidades para desenvolver o brincar utilizando formas criativas por meio da imaginação, o que contribuiu para a interação com outros indivíduos.

Vieira, Matos, Ivo e Carneiro (2010) verificaram os efeitos do brincar no hospital para crianças com câncer, nas diferentes fases de tratamento. As autoras estabeleceram relações entre o brincar da criança na fase crítica de tratamento (recidiva, inchaço, queda de cabelo) e na fase de manutenção, quando a criança se submetia ou não ao exame de punção lombar. Foram apresentados diversos brinquedos e jogos infantis, em sessões registradas por meio de videogravações. Com o auxílio da observação participante, foi possível verificar o interesse das crianças para o brincar, a interação nas brincadeiras e no jogo social, independentemente da fase de tratamento. Esses dados permitem compreender as contri- 
buições do brincar para a criança que vivencia etapas relacionadas ao tratamento da doença crônica.

Silva, Borges e Mendonça (2010) investigaram de que maneira as atividades lúdicas realizadas pelos profissionais de educação física podem contribuir para a criança com câncer. Após o desenvolvimento de intervenções lúdicas com diversos brinquedos e jogos, foram realizadas entrevistas com as crianças e os acompanhantes. As crianças destacaram os benefícios do brincar para a promoção da alegria, enquanto os acompanhantes relataram a importância do brincar para o desenvolvimento da espontaneidade. A partir desses dados, verifica-se que existem congruências nos relatos dos participantes da pesquisa.

Silva (2006) investigou as percepções de profissionais de saúde acerca do brincar das crianças hospitalizadas com câncer. Para os participantes, as atividades lúdicas auxiliaram o processo de recuperação da criança e permitiram reduzir a apatia, promovendo interação social, momentos de satisfação e mudanças positivas no humor. Os dados evidenciaram que o brincar representa um instrumento importante para o desenvolvimento de habilidades adaptativas da criança com câncer.

\section{Vivências de crianças com câncer}

As pesquisas acerca do assunto focalizam a perspectiva da criança, utilizando o brinquedo terapêutico para investigar as perdas simbólicas, os significados relacionados à morte, a experiência diante dos procedimentos técnicos utilizados no tratamento e os sentidos atribuídos a ser criança com câncer.

No estudo de Almeida (2005), procurou-se compreender de que maneira as crianças com câncer vivenciam as perdas simbólicas do processo de hospitalização e atribuem significados à morte. Nas sessões de brinquedo terapêutico, verificou-se que o distanciamento das pessoas do ambiente familiar e social e as mudanças na rotina de vida diária representaram as principais perdas. Na dramatização de procedimentos realizados pela equipe de saúde, as crianças destacaram a morte temporária dos familiares e profissionais do setor. Verificou-se pouca receptividade das crianças para os brinquedos que se referiam a objetos de uso hospitalar, tais como soro, seringa e estetoscópio. Os dados indicaram que, por meio do brincar, a criança hospitalizada com câncer compreende as experiências vivenciadas nesse ambiente e atribui significados para a superação das dificuldades.

A pesquisa de Ribeiro, Coutinho, Araújo e Souza (2009) buscou analisar a vivência da criança com câncer portadora de cateter. Foram realizadas sessões de brinquedo terapêutico com bonecos que representavam a família e os profissionais de saúde, objetos da unidade hospitalar e diversos brinquedos. A análise de dados evidenciou que as crianças vivenciavam sensações de dor, medo e desconforto que estão relacionadas com o risco de infecção, mas os momentos destinados ao brincar promoveram a satisfação e a expressão de sentimentos relacionados ao processo de hospitalização. As crianças solicitaram a presença de brinquedos e iniciaram dramatizações que permitiram facilitar o enfrentamento da vivência de hospitalização. De forma específica, o brincar contribui para a criança com câncer expressar percepções acerca de procedimentos invasivos.

O estudo de Melo e Valle (2010) objetivou investigar o sentido de ser criança com câncer. As autoras utilizaram o espaço da brinquedoteca para favorecer a expressão das crianças acerca de suas vivências. Os relatos das crianças e as observações das sessões foram registrados em um diário de campo. A angústia de estar doente e a insatisfação com as características e regras do ambiente hospitalar representaram os principais temas que foram identificados. No que se refere ao brincar, as crianças demonstraram interesse e construíram dramatizações com os personagens infantis, evidenciando as dificuldades enfrentadas para o tratamento do câncer. Segundo as autoras, o brincar contribui para o desenvolvimento integral da criança, sendo necessário valorizar a aplicação desse recurso nos ambientes de saúde.

\section{Construção de projetos lúdicos}

As pesquisas não só evidenciam os efeitos positivos de projetos lúdicos para a criança com câncer, como também apresentam a metodologia e os recursos utilizados para a implantação de intervenções lúdicas no ambiente hospitalar, além de expor a opinião de crianças, acompanhantes e equipe de saúde.

Vieira e Carneiro (2006) desenvolveram um projeto lúdico para crianças com câncer, em que foram utilizados diversos brinquedos, de forma individual ou coletiva. As autoras investigaram os conteúdos das brincadeiras de faz-de-conta das crianças, a partir dos 
relatórios acerca do brincar. Foram identificados temas específicos relacionados ao adoecimento, tais como consulta, morte, ambulância, ao lado da prevalência de temas genéricos que incluíam cenas domésticas. As brincadeiras das crianças com câncer relacionavam situações de morte, o tratamento da quimioterapia e os efeitos de queda de cabelo. Os dados mostraram que, quando a criança compreende sua experiência de adoecimento e hospitalização, isso contribui para o enfrentamento das situações.

Costa-Júnior, Coutinho e Ferreira (2006) investigaram os efeitos de um programa de recreação planejado para crianças com câncer. Participaram da amostra crianças de diferentes faixas etárias. Nas sessões de recreação, foram selecionados recursos lúdicos gerais que relacionavam diversas modalidades de jogos e miniaturas de brinquedos, bem como materiais específicos sobre temáticas do ambiente hospitalar, tais como maleta de médico e dominó sobre o corpo humano. 0 comportamento das crianças foi registrado, e a análise de dados indicou que o acúmulo de sessões aumentava a iniciativa da criança no brincar, com o desenvolvimento da interação social, a manifestação de expressões verbais e a participação efetiva nas brincadeiras. Os autores evidenciaram a importância das intervenções lúdicas para as crianças nos ambientes de saúde, o que permite compreender aspectos relacionados à adaptação e enfrentamento da doença e tratamento.

A construção do projeto lúdico "Diversão em Movimento", no serviço de oncologia pediátrica, representou a temática da pesquisa de A.M. Pedrosa, Monteiro, Lins, F. Pedrosa e Melo (2007). Foram selecionados livros, brinquedos, papéis e lápis coloridos. Esses materiais lúdicos foram organizados em um carro de curativos que foi adaptado para percorrer as enfermarias da instituição hospitalar, visando proporcionar momentos de diversão de forma dinâmica para as crianças e acompanhantes. Os autores destacaram que os participantes desenvolveram a interação social expressando satisfação, aspectos positivos para as vivências no hospital.

Jesus, Borges, Pedro e Nascimento (2010) procuraram conhecer a opinião de acompanhantes de crianças com câncer, acerca do projeto lúdico denominado "Quimioteca". Trata-se de um espaço para a realização da quimioterapia, que integra brinquedos, jogos, livros e músicas, além de ilustrações coloridas nas paredes para tornar o ambiente agradável. Os participantes 570 destacaram a influência positiva do ambiente para a qualidade do tratamento e a satisfação da criança, o que possibilita a distração acerca das sensações dolorosas, além de auxiliar a criança em relação ao tempo que permanece no hospital. Para os autores, a possibilidade de as crianças com câncer brincarem durante a realização da quimioterapia representa uma estratégia relevante para a humanização da assistência hospitalar. Dessa forma, as ações destinadas para crianças e acompanhantes precisam considerar o cuidado integral à vida do ser humano.

\section{Modalidades do brincar no hospital}

Considerando que as atividades lúdicas integram diversas modalidades, tais como brincar, jogar e cantar, verificou-se que as pesquisas investigaram a utilização da música no ambiente hospitalar e as repercussões psicológicas para a criança com câncer.

Barrera, Rykov e Doyle (2002) buscaram verificar os efeitos da música nas crianças hospitalizadas com câncer. As sessões com música foram realizadas no hospital e, em seguida, foi apresentado numa folha um conjunto de expressões faciais para a criança identificar os sentimentos. Também os acompanhantes avaliaram a atuação da criança no brincar. No que se refere às crianças, ocorreu melhoria significativa nas avaliações sobre seus sentimentos. Da mesma forma, os acompanhantes avaliaram positivamente as intervenções musicais com crianças em idade pré-escolar. A análise de dados indicou os benefícios da música para o bem-estar da criança hospitalizada com câncer.

Robb et al. (2008) também avaliaram um programa de intervenção que utilizou a música para crianças hospitalizadas com câncer. Os pesquisadores, por meio da metodologia experimental, selecionaram grupos de crianças para avaliar níveis comportamentais: o primeiro grupo participava das músicas cantando ou dançando, o segundo apenas escutava as músicas, o terceiro escutava o áudio dos livros de histórias infantis. As sessões foram gravadas, e as análises indicaram que o primeiro grupo apresentou maior índice de comportamentos relacionados à iniciação e envolvimento, se comparado aos outros grupos. Os autores destacaram a importância das intervenções com música para a mudança no humor e o desenvolvimento de atividades motoras. Acredita-se que a utilização de diferentes recursos lúdicos no hospital contribui para a ampliação das pesquisas e valorização do universo infantil. 


\section{Discussão}

Na análise da produção científica acerca do brincar da criança com câncer no hospital, verificou-se que as pesquisas investigaram os possíveis benefícios dos recursos lúdicos e evidenciaram dados positivos. Apenas o estudo de Gariépy e Howe (2003) indicou pouca adesão das crianças, o que foi relacionado com os níveis de stress, mas outros estudos indicam novas perspectivas (Vieira et al., 2010).

Observou-se a participação de acompanhantes e equipe de saúde nas pesquisas, por considerar que essas pessoas auxiliam a criança hospitalizada. A família da criança com câncer vivencia situações de crise diante do direcionamento de novos papéis para estabelecer a rede de apoio (Pedrosa \& Valle, 2000; Ortiz, 2003). A criança e o núcleo familiar compartilham a angústia gerada pelo tratamento invasivo, fazendo com que a acompanhante (representada pela genitora) permaneça no hospital durante um período indeterminado, de modo que os demais integrantes precisam reorganizar a dinâmica familiar. Diante das mudanças na sua vida, a criança com câncer, de qualquer idade, percebe que alguma coisa grave está acontecendo, e tem necessidade de identificar essa situação (Menezes, Passareli, Drude, Santos \& Valle, 2007). O desenvolvimento de atividades lúdicas representa uma possibilidade para a criança expressar suas vivências, minimizar os efeitos do processo de hospitalização, reestruturar as experiências traumáticas e fortalecer os vínculos afetivos.

Nas pesquisas, identificou-se a receptividade das crianças para o brincar, assim como o interesse para desenvolver a espontaneidade e a interação social. Os efeitos positivos dos projetos lúdicos no hospital permitem evidenciar as contribuições para a qualidade de vida da criança e sua família. Kohlsdorf e Costa-Júnior (2008) destacaram a importância da participação do psicólogo e outros profissionais de saúde no desenvolvimento de atividades lúdicas para a criança com câncer, visando fortalecer o apoio para o enfrentamento do período de hospitalização. O brincar no hospital promove o resgate da concepção de vida, o estabelecimento de relações sociais e a integração da equipe de saúde na assistência à criança e ao acompanhante.

De acordo com Silva (2006), o brincar estimula o desempenho criativo e, para compreender a vivência da criança com câncer, torna-se necessário identificar suas características pessoais e a dinâmica estabelecida nas relações intergrupais, o que contribui para a valorização da pessoa humana. Assim, a criança sente-se acolhida e respeitada pelas outras pessoas enquanto brinca, quando no ambiente hospitalar existe a possibilidade de desenvolver suas potencialidades.

\section{Considerações Finais}

No que se refere às pesquisas que foram consultadas, é importante destacar que o maior número de publicações foi identificado nas revistas nacionais, o que permite ressaltar o interesse dos autores brasileiros para o estudo do brincar no hospital, da criança com câncer.

Os dados das pesquisas indicam os avanços ocorridos na investigação dessa temática no setor de oncologia infantil, os benefícios do brincar para a recuperação da criança e a avaliação positiva de acompanhantes e equipe de saúde, o que possibilita evidenciar a ação terapêutica das intervenções lúdicas.

De outro lado, o baixo índice de produções internacionais representou a principal limitação deste estudo no sentido de apresentar o panorama atual de publicações. Estima-se que provavelmente algumas revistas estejam em processo de indexação eletrônica.

Por fim, recomenda-se a continuidade das pesquisas para o aprimoramento de estudos na área da psicologia pediátrica, visando analisar questões referentes à inserção do brincar no hospital.

\section{Referências}

Almeida, F. A. (2005). Lidando com a morte por meio do brincar: a criança com câncer no hospital. Boletim de Psicologia, 55 (123), 149-167.

Bardin, L. (1979). Análise de conteúdo. Lisboa: Edições 70.

Barrera, M. E., Rykov, M. H., \& Doyle, S. L. (2002). The effects of interactive music therapy on hospitalized children with cancer: a pilot study. Psycho-Oncology, 11 (5), 379-388.

Borges, E. P., Nascimento, M. D. S. B., \& Silva, S. M. M. (2008). Benefícios das atividades lúdicas na recuperação de crianças com câncer. Boletim Academia Paulista de Psicologia, 18 (2), 211-221.

Bomtempo, E., Hussein, C. L., \& Zamberlan, M. A. T. (1986). Psicologia do brinquedo: aspectos teóricos emetodológicos. São Paulo: Nova Stella.

Bomtempo, E. (1987). Aprendizagem e brinquedo. São Paulo: EPJ. 
Borges, A. D. V. S. , Silva, E. F., Mazer, S. M. , Toniollo, P. B.; Valle, E. R. M., \& Santos, M. A. (2006). Percepção da morte pelo paciente oncológico ao longo do desenvolvimento. Psicologia em Estudo, 11 (2), 361-369.

Carvalho, A. M., \& Begnis, J. G. (2006). Brincar em unidades de atendimento pediátrico: aplicações e perspectivas. Psicologia em Estudo, 11 (1), 109-117.

Chiattone, H. B. C. (2003). A criança e a hospitalização. In V. A. A. Camon (Org.), A psicologia no hospital (2a ed., pp.23-99). São Paulo: Pioneira Thomson Learning.

Costa-Júnior, A. L., Coutinho, S. M. G., \& Ferreira, R. S. (2006). Recreação planejada em sala de espera de uma unidade pediátrica: efeitos comportamentais. Paidéia, 16 (33), 111-118

Dias, R. R., Baptista, M. N., \& Baptista, A. S. D. (2003). Enfermaria de pediatria: avaliação e intervenção psicológica. In R. R. Dias, M. N. Baptista \& A. S. D. Baptista (Orgs.), Psicologia hospitalar: teoria, aplicações e casos clínicos (pp.53-73). Rio de Janeiro: Guanabara.

Gariépv, N., \& Howe, N. (2003). The therapeutic power of play: examining the play of young children whith leukaemia. Child: Care, Health and Development, 29 (6), 523-537.

Instituto Nacional do Câncer. (2008). Informações gerais acerca do câncer. Recuperado em setembro 15, 2008, disponível em <http://www2.inca.gov.br>.

Jesus, I. Q., Borges, A. L. V., Pedro, I. C. S., \& Nascimento, L. C. (2010). Opiniões de acompanhantes de crianças em quimioterapia ambulatorial sobre uma quimioteca no município de São Paulo. Acta Paulista de Enfermagem, 23 (2), 175-180.

Kohlsdorf, M., \& Costa-Junior, A. L. (2008). Estratégias de enfrentamento de pais de crianças em tratamento de câncer. Estudos de Psicologia (Campinas), 8 (1), 417-429.

Leontiev, A. N. (1998). Uma contribuição à teoria do desenvolvimento da psique infantil. In L. S. Vigotski, A. R. Luria \& A. N. Leontiev (Orgs.), Linguagem, desenvolvimento e aprendizagem (pp.119-142). São Paulo: Ícone.

Lindquist, I. (1993). A criança no hospital: terapia pelo brinquedo. São Paulo: Scritta.

Melo, L. L., \& Valle, E. R. M. (2010). A brinquedoteca como possibilidade para desvelar o cotidiano da criança com câncer em tratamento ambulatorial. Revista da Escola de Enfermagem da USP, 44 (2), 517-525.

Menezes, C. N. B., Passareli, P. M., Drude, F. S., Santos, M. A., \&Valle, E. R. M. (2007). Câncer infantil: organização familiar e doença. Revista Mal-Estar e Subjetividade, 7 (1), 191-210.

Motta, A. B., \& Enumo, S. R. F. (2004). Câncer infantil: uma proposta de avaliação das estratégias de enfrentamento da hospitalização. Estudos de Psicologia (Campinas), 21 (3), 193-202.

Oliveira, S. S. G., Dias, M. G. B. B., \& Roazzi, A. (2003). O lúdico e suas implicações nas estratégias de regulação emocional em crianças hospitalizadas. Psicologia: Reflexão e Crítica, 16 (1), 1-13.

Ortiz, M. C. M. (2003). À margem do leito: a mãe e o câncer infantil. São Paulo: Arte \& Ciência.

Pedrosa, A. M., Monteiro, H., Lins, K., Pedrosa, F., \& Melo, C.

572 (2007). Diversão em movimento: um projeto lúdico para crianças hospitalizadas no Serviço de Oncologia Pediátrica do Instituto Materno Infantil Prof Fernando Figueira, IMIP. Revista Brasileira Saúde Materno Infantil, 7(1), 99-106.

Pedrosa, C. M., \& Valle, E.R.M. (2000). Ser irmão de criança com câncer: um estudo compreensivo. Pediatria, 22 (2), 185-194.

Pérez-Ramos, A. M. Q. (2006). O ambiente na vida da criança hospitalizada. In E. Bomtempo, E. G. Antunha \& V. B. Oliveira (Orgs.), Brincando na escola, no hospital, na rua (pp.111-126). Rio de Janeiro: Wak.

Ribeiro, C. A., \& Angelo, M. (2005). O significado da hospitalização para a criança pré-escolar: um modelo teórico. Revista da Escola de Enfermagem da USP, 39 (4), 391-400.

Ribeiro, C. A., Coutinho, R. M., Araújo, T. F., \& Souza, V. S. (2009). Vivenciando um mundo de procedimentos e preocupações: experiência da criança com Port-a-Cath. Acta Paulista de Enfermagem, 22 (n. especial), 935-941.

Robb, S. L., Watanabe, M., Monahan, P., Azzouz, F., Stouffer, J. W., Ebberts, A., et al. (2008). Randomized controlled trial of the active music engagement (AME) intervention on children with cancer. Psycho-Oncology, 17 (7), 699-708.

Silva, S. M. M. (2006). Atividades lúdicas e crianças hospitalizadas por câncer: o olhar dos profissionais e das voluntárias. In E. Bomtempo, E. G. Antunha \& V. B. Oliveira (Orgs.), Brincando na escola, no hospital, na rua (pp.127-142). Rio de Janeiro: Wak.

Silva, S. M. M., Borges, E. P., \& Mendonça, F. M. A. (2010). O brincar para as crianças hospitalizadas e suas mães. In A. M. Q. Pérez-Ramos \& V. B. Oliveira (Orgs.), Brincar é saúde: o lúdico como estratégia preventiva (pp.101-132). Rio de Janeiro: Wak.

Silva, L. F., Cabral, I. E., \& Christofell, M. M. (2008). O brincar na vida do escolar com câncer em tratamento ambulatorial: possibilidades para o desenvolvimento. Revista Brasileira Crescimento e Desenvolvimento Humano, 18 (3), 275-287.

Valle, E. R. M. (2001). Possibilidades de atuação em Psicooncologia pediátrica. In E. R. M. Valle (Org.), Psico-oncologia pediátrica (pp.77-85). São Paulo: Casa do Psicólogo.

Vieira, T., \& Carneiro, M. (2006). O brincar na sala de espera de um ambulatório pediátrico: possíveis significados. In E. Bomtempo, E. G. Antunha \& V. B. Oliveira (Orgs.), Brincando na escola, no hospital, na rua (pp.75-110). Rio de Janeiro: Wak.

Vieira, T., Matos, D. P., Ivo, D. M. T. S., \& Carneiro, M. S. (2010). A criança com leucemia, o seu tratamento e o brincar. In A. M. Q. Pérez-Ramos \& V. B. Oliveira (Orgs.), Brincar é saúde: o lúdico como estratégia preventiva (pp.133-170). Rio de Janeiro: Wak.

Vigotski, L. S. (1998). A formação social da mente. São Paulo: Martins Fontes.

Yamaguchi, N. H. (1994). O câncer na visão da oncologia. In M. M. M. J. Carvalho (Org.), Introdução à psiconcologia (pp.21-32). Campinas: Editorial Psy.

Recebido em: 5/1/2009

Versão final reapresentada em: 2/12/2010

Aprovado em: 27/6/2011 